\title{
Cognitive Power Adjustment for OFDM-Based Wireless Communication System: An Empirical Study
}

\author{
W. Hashim, A. F. Ismail, N. M. Anas, H. A. M. Ramli, and K. Abdullah
}

\begin{abstract}
In this paper, we describe an empirical study on the impact of throughput performance when transmission power is adjusted. This experiment was conducted in an office environment for an OFDM-based wireless communication system such as WiFi 802.11g protocol network. We present a power selection mechanism based on intuitively learning one of the attributes which then triggers the power adjustment. We show that client's similar throughput performance can be obtained at reduced transmission power thus prevent an access point from maximizing power unnecessarily. Our cognitive power adjustment method is potentially useful in extremely dense wireless networks deployment since it is able to prevent from any interference and also to reduce energy consumption of the devices. Our evaluation of this mechanism can reduce the power to $5 \mathrm{~dB}$ while maintaining the same level of throughput.
\end{abstract}

Index Terms-Cognitive selection mechanism, transmit power control, intelligent power adjustment, interference.

\section{INTRODUCTION}

Current technologies such as IEEE 802.11, LTE, WiMAX and near-future such as LTE-Advanced, IEEE 802.11 ac/ad/af and High Efficiency WLAN - HEW have been driven by the incessantly demand of mobile users for more capacity and better coverage. The more sustainable solution to the $5 \mathrm{G}$ promise of true ubiquitous mobile broadband is to deploy very dense wireless networks, which is called DenseNets [1]. Some of the relevant issues already identified in the DenseNets scenarios include the nonlinear characteristics of the achieved throughput and energy consumption. As an increased number of cells are being deployed, the effect of the interference becomes dominant, resulting on an overall throughput which is not linear with the number of cells. Similar nonlinear effects on energy efficiency have been observed due to the need for the equipment to be continuously serving high demand applications [1].

In close proximity cell arrangement, transmission in overlapping cells tends to interfere with each other. This will adversely impact the aggregate wireless network throughput and thus the quality of applications such as multimedia streaming [2]. In cellular network, smaller cell sizes with lower transmit power leads to the higher overall network

Manuscript received February 15, 2014; revised April 19, 2014.

W. Hashim and N. M. Anas are with the Malaysia National ICT Research and Development, MIMOS Berhad, Kuala Lumpur, 570000 Malaysia (e-mail: wahidah.hashim@ mimos.my,nuzli.anas@mimos.my).

A. F. Ismail, H. A. M. Ramli, and K. Abdullah are with the International Islamic University of Malaysia, Gombak 50728 Kuala Lumpur, Malaysia (e-mail: af_ismail@iium.edu.my, hadibahmr@iium.edu.my, khaizuran@iium.edu.my). capacity. The objective of Transmit Power control (TPC) on a wireless device (AP or STA) is to use minimum transmit power while meeting the requirements for throughput and packet loss rate. TPC helps reduce interference with other devices, improve channel reuse, and eventually increase the overall capacity in wireless networks. In addition, TPC also helps conserve energy and improve battery life of mobile devices [2]. However intelligent algorithms are required to adapt transmit power in a practical and distributed way to achieve improvement in performance. In practice, system networking company such as Cisco incorporates its TPC module in specific access point's resource management to reduce power with the assistance of three more nearby access points [3]. Power increment is not programmed in this TPC but it is covered in "coverage" hole algorithm. In each decrement or increment of power there is a limit that has been set in the device as an example minimum power reduction is at $14 \mathrm{~dB}$. Higher power level settings may be constrained by local regulatory requirements and AP capabilities.

Another TPC example in practice is by Aruba Networks. The control in power is implemented based on the average link whether it is line-of-sight (LOS) or non-line-of-sight (NLOS) between AP and clients. APs on a ceiling often have good line of sight between them, which will cause adaptive resource management (ARM) to decrease their power to prevent interference. However, if the wireless clients down on the floor do not have such a clear line back to the AP, client could end up with coverage gaps [4]. The intelligence built in Cisco requires involvement of other nearby APs before decision can be made. In the event that one of the APs is downed, the TPC algorithm will not be able to function properly. On the other hand, the power control in Aruba AP is limited to position of the AP and the clients. Knowing that clients are mostly scattered and blocked by furniture, issues of coverage gap can happen. We have proposed a new technique of controlling the transmission power by adjusting it based on the assessment of the surrounding environment using parameter such as received signal strength (RSS). This is adopted since it is the simplest parameter to evaluate for empirical research which involved development such algorithm in the hardware. However, other parameters will be considered in the future as progresses. Such intelligent TPC, we coin the term as 'Cognitive Power Adjustment'.

The rest of this paper is organized as follows. Section II describes cognitive power adjustment, concept and flow chart. Section III covers brief explanation on the system architecture and models. Experimental setup and system parameter are elaborated in Section IV followed by experimental results in Section V. Finally, Section VI presents the concluding remarks. 


\section{Cognitive Power AdJustment}

This section described on our proposed cognitive power adjustment which was developed and incorporated in an access point for proof-of-concept purpose.

\section{A. Concept of Cognitive Power Adjustment}

The concept of cognitive power adjustment was originated from our work on cognitive selection mechanism (CSM) [5]-[8]. We have extended related work in CSM to power adjustment application. An aspect of cognitive sciences deployed is the artificial intelligence (AI) to smartly choose and decide for best element of selection. This is achieved by implementing such components in an ingenious algorithm that capable of controlling the element selection at the physical layer of a communication device. The massively discussed Internet-of Things (IoT) [9], demands for future generation network $(5 \mathrm{G}, 6 \mathrm{G})$ to become smarter, able to learn, to sense the environment and energy efficient. Such requirements obviously need cognitive intelligence in each communication device. Fig. 1 depicts the proposed cognitive power adjustment.

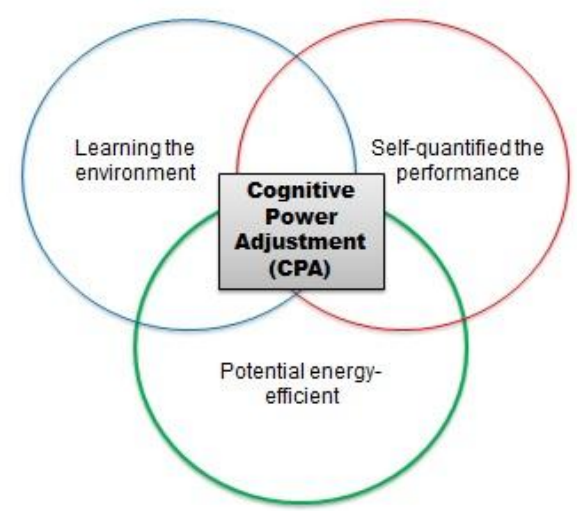

Fig. 1. Proposed concept of Cognitive Power Adjustment (CPA)

\section{B. Flow Chart}

The proposed cognitive power adjustment algorithm able to scan, analyze and evaluate the given parameters for finding the most suitable power of an AP. It is through scanning and analyzing process that this concept manipulates the learning of the network environment in its power decision. Fig. 2 highlights the flow diagram of CPA which is targeted at AP. It begins with measuring the current RSS and device transmission power. Once captured, the current throughput between local AP and randomly select client is measured. That information is stored within a temporary database. Transmission power is adjusted based on some predefined rules. This is considered as temporary power. In our proposal we have set as such;

1) Low: RSSI < $-75 \mathrm{~dB}$, increase transmission power to $27 \mathrm{~dB}$.

2) High: RSSI > $-50 \mathrm{~dB}$, reduce transmission power to $15 \mathrm{~dB}$

3) Medium: $-75<\mathrm{RSSI}<-50 \mathrm{~dB}$, adjust transmission power to $20 \mathrm{~dB}$.

Our selection on this value and range is based on the preliminary experiment in the laboratory and also the limitation power given by hardware manufacturer.

Once the temporary transmission power (TTP) is self-configured in the communication device, another new evaluation of throughput is assessed. Should the TTP improve the throughput; the system will decide to retain the TTP value. Otherwise, maintain with the original power. In our study, the update of this power is conducted on every 120 minutes and the time is specified as the interval of time for the program to repeats its evaluation and selection of the best power. However, the selected time interval as well as the power rules can be changed according to preferred condition and location.

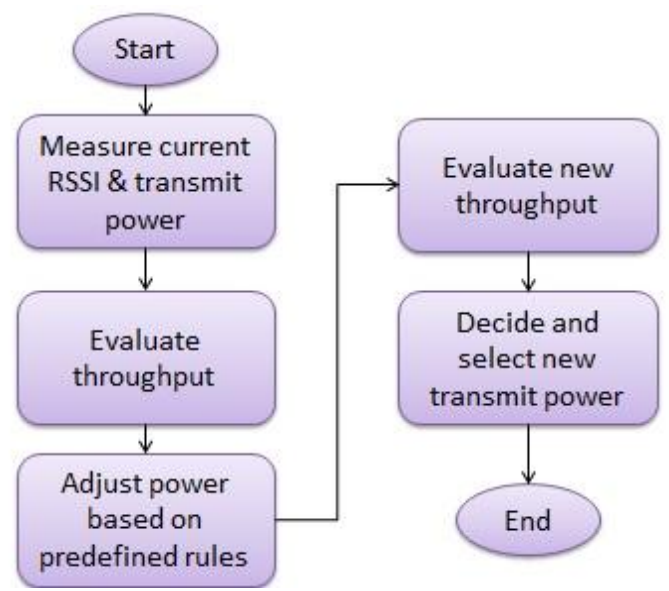

Fig. 2. Flow chart of cognitive power adjustment for an access point.

\section{SYSTEM ARCHITECTURE}

\section{A. System Architecture and Scenario}

In this section, the system architecture and model are described. As mentioned in the introduction section, such CPA is targeted to be applied in dense wireless networks (DensNet). Nonetheless for the purpose of illustration, we only shows network with three APs. Fig. 3 depicts the scenario whereby no CPA is incorporated in the conventional AP.

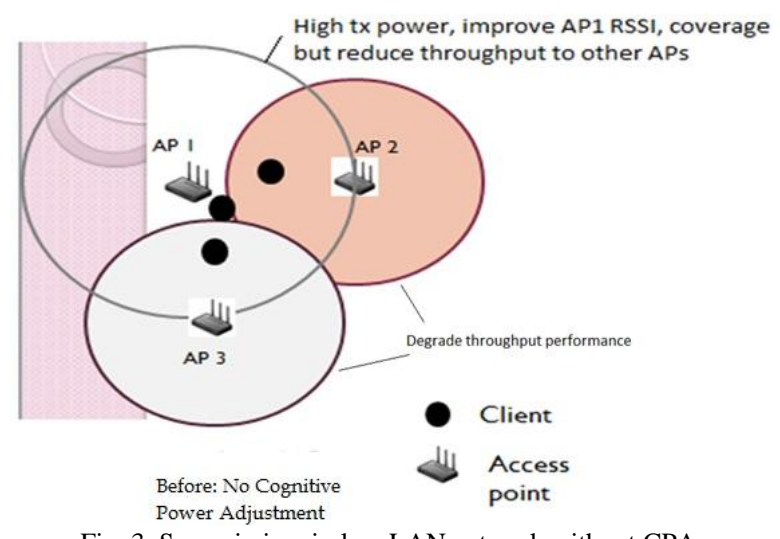

Fig. 3. Scenario in wireless LAN network without CPA.

In Fig. 3, if the AP has not control on its transmission power, this may cause interference to the nearby AP in the neighborhood. Although high transmit power usually perceived can improve RSSI and coverage hence throughput, the impact to other AP performances in the whole network can be negative. Situations whereby most clients are near to the AP, can tolerate some transmission power reduction while still maintaining good RSSI. For the clients at the edge coverage, if knowing connecting to the AP gives a bad RSSI, 
the client should be connected to other alternative APs. If this is exercised, a harmony, unselfish and stable wireless LAN network can be achieved. Hence, the incorporation of our proposed CPA can be illustrated as in Fig. 4.

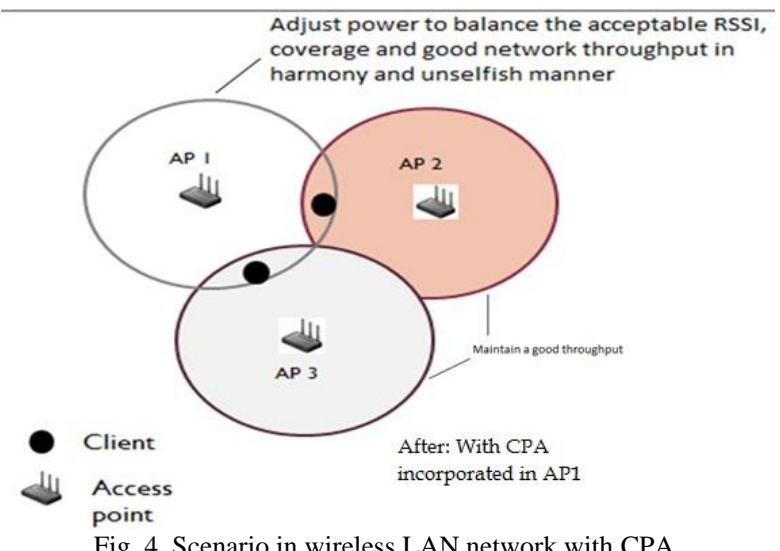

\section{B. System Model}

On the other hand, Fig. 5 illustrates the system model which consists of measurement unit, decision unit, and evaluation unit as well as the threshold model. The whole units are built in the algorithm and later embedded in the hardware.

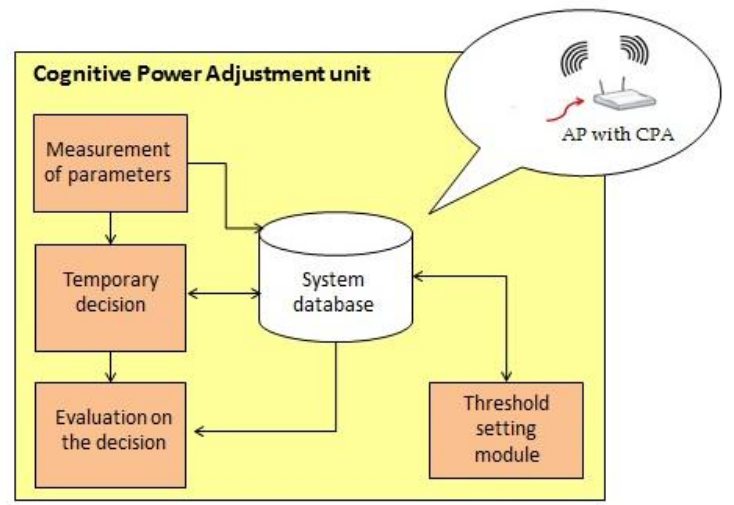

Fig. 5. System model for cognitive power adjustment.

\section{EXPERIMENTAL SETUP}

\section{A. System Setup}

In this section, the experimental setup of the proposed algorithm is elaborated. CPA was developed in our laboratory based on an open source programming language such as Voyage Linux system. Once completed, the algorithm was embedded into a development board from PC Engine namely Alix 3D2 model as shown in Fig. 6. This is the main processor for the AP.

\section{B. System Parameters}

Our system parameters for laboratory experiment are listed in Table I. We have used WLAN frequency at $2.4 \mathrm{GHz}$ band following the IEEE 802.11 protocols.

For the empirical study, the completed proof-of-concept $(\mathrm{PoC})$ together with the development board as shown in Fig. 7 , was tested in two environments. One was in the laboratory shield room (Fig. 8) and one is in the office environment. For shield, the PoC was tested and verify to ensure the algorithm performance at its best.

TABLE I: SYSTEM PARAMETERS

\begin{tabular}{|l|l|}
\hline Parameter & Description \\
\hline Spectrum band & WiFi $-2.4 \mathrm{GHz}$ \\
\hline Operating system & Ubuntu 12.04 LTS \& Voyage \\
\hline Channel tested & Uncongested available channels \\
\hline
\end{tabular}

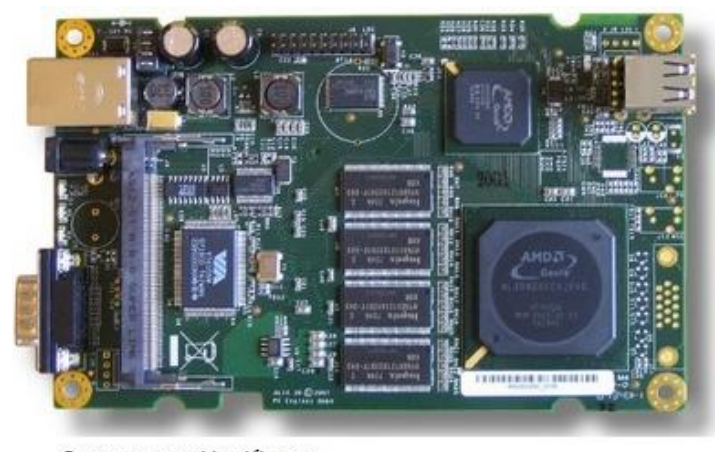

Source: www.i4-wifi.com

Fig. 6. Alix 3D2 model from PC engine.

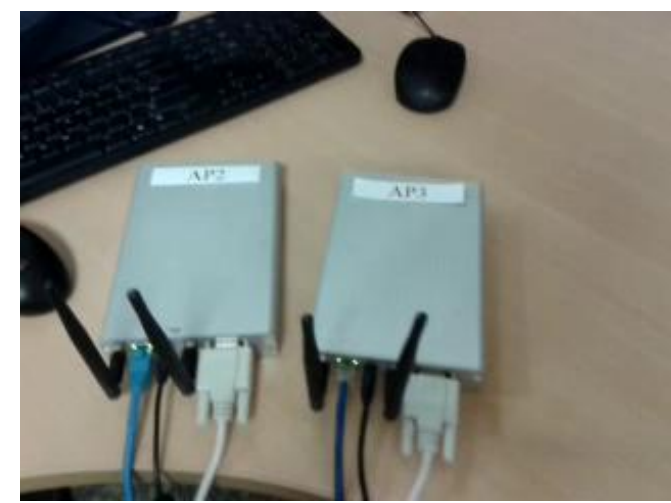

Fig. 7. Access points used in the laboratory PoC @2014 MIMOS Berhad.

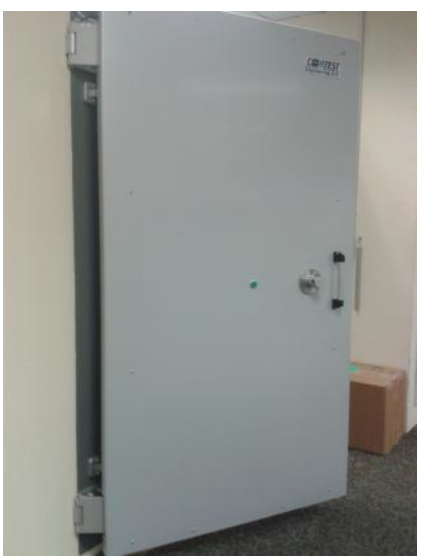

Fig. 8. Shield room for laboratory experiment @2014 MIMOS Berhad.

\section{EXPERIMENTAL RESULTS}

This section provides itemized analyses on the cognitive power adjustment algorithm that had been developed. It is also presents detailed analyses on each particularized throughput that had been accumulated for specific measurement period. In Fig. 9, when transmission power is adjusted between $15 \mathrm{dBm}$ to maximum value allowed i.e. $27 \mathrm{dBm}$, the throughput at $27 \mathrm{dBm}$ can also be achieved at $15 \mathrm{dBm}$. In fact it is better than at 20 or $25 \mathrm{dBm}$. On average the throughput can be calculated as $1.5 \mathrm{Mbps}$ and this is only $0.5 \mathrm{Mbps}$ different from the maximum value of $2 \mathrm{Mbps}$ Below than $10 \mathrm{dBm}$, the descending performance of data rate 
is quite significant hence that is the reason why in our experiment we capped the lowest transmission power at $15 \mathrm{dBm}$ since at this power level, throughput value can still be maintained.

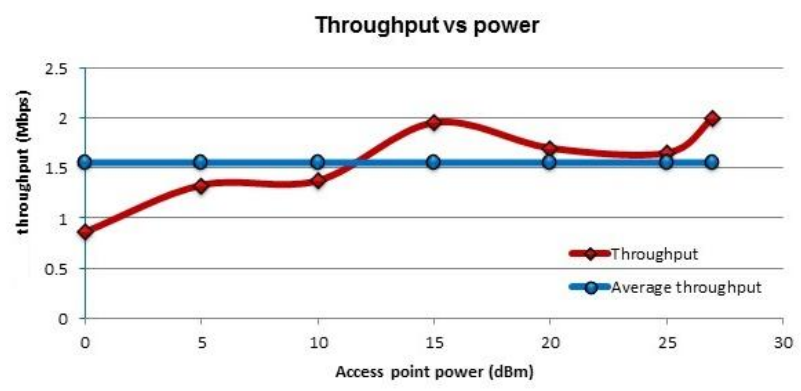

Fig. 9. Throughput performance over variation of transmit powers.

The next Fig. 10 presents the impact of throughput performance on other APs when transmission power of local AP is reduced. It was observed that when the third AP is reduced its power, there is significant changes to the throughput of other neighboring APs. As an example, when AP3 was set its power to $20 \mathrm{dBm}$, the average throughput obtained is about $3.558 \mathrm{Mbps}$ and when AP3 reduced its power to $15 \mathrm{dBm}$ the throughput of AP1 improved to $5.18 \mathrm{Mbps}$. This significant improvement is about $46 \%$.

On the other hand, the improvement gained by AP2 when the same condition applied was only $25 \%$. The average throughput of AP2 when AP3 power was $20 \mathrm{dBm}$ is $4.332 \mathrm{Mbps}$ and for $15 \mathrm{dBm}$ the throughput was obtained on average at $5.412 \mathrm{Mbps}$. Nonetheless, there is significant improvement in both APs. The reason for little improvement maybe due to the distance between AP3 and AP2 whereby here was not much impact on the power when the distance is far or non-overlapping of coverage from AP3.

\section{Power Impacts on Throughput}

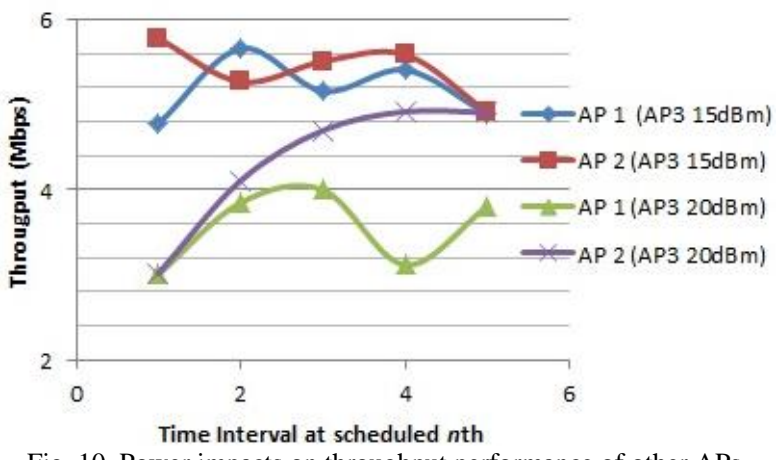

Fig. 10. Power impacts on throughput performance of other APs.

\section{CONCLUSION}

As the conclusion, our proposed cognitive power adjustment gives a significant performance improvement to the throughput on the local APs as well as the neighboring APs. A maximum improvement of $46 \%$ in the throughput shows that when the transmit power is controlled cooperatively, the entire network can benefit the improvement thus serving the clients better. By reducing the power, we can ensure that there is a potential energy efficient element in the proposed CPA. Since measuring the energy efficiency requires more detailed studies, we will take that as our future works.

\section{REFERENCES}

[1] High-Density Wi-Fi Design Principles, Aerohive Networks White Paper, 2012.

[2] H. Viswanathan, "Adaptive transmit power control based on signal strength and frame loss measurements for WLANs," MSc. dissertation, Dept. Elect. \& Comp. Eng., New Brunswick Rutgers Univ., New Jersey, NJ, 2009.

[3] Transmit Power Control Algorithm, Cisco Radio Resource Management Under Unified Wireless Networks, Doc ID: 71113, May 2010.

[4] Configuring Adaptive Radio Management (ARM) Profiles and Settings, Aruba User Guide, Aruba Networks Inc, Sunnyvale, CA, 2008, pp. 4-6.

[5] W. Hashim, A. F. Ismail, S. Dzulkifly, and N. A. A. Ghafar, "Cognitive Selection Mechanism for Indoor Propagation," International Journal of Computer and Comm. Engineering, vol. 2, no. 4, pp. 433-438, July 2013.

[6] W. Hashim, A. F. Ismail, S. Dzulkifly, and N. A. A. Ghafar, "Cognitive Selection Mechanism Performance in IEEE 802.11 WLAN," International Journal of Computer and Comm. Engineering, vol. 2, no. 4, pp. 477-481, July 2013.

[7] W. Hashim. A. F. Ismail, S. Dzulkifly, and N. A. A. Ghafar, "Performance Analysis of Cognitive Selection Mechanism for WLAN System," presented at the IEEE International Symposium on Antennas and Propagation and USNC-URSI National Radio Science Meeting, Orlando, FL, July 7-13, 2013.

[8] W. Hashim. A. F. Ismail, S. Dzulkifly and N. A. A. Ghafar, "An Empirical Study of Cognitive Selection Mechanism Deployment in Wireless Communication," presented at the IEEE International Symposium on Antennas and Propagation and USNC-URSI National Radio Science Meeting, Orlando, FL, July 7-13, 2013.

[9] A. McEwen and H. Cassimally, Designing the Internet of Things, $1^{\text {st }}$ ed., Chichester, U. K.: Wiley, 2014, p. 308.

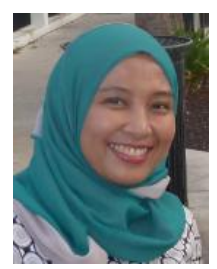

Wahidah Hashim received her bachelor degree in information technology, business management and language from University of York, UK in 1999. She then pursued her MSc in Multimedia Technology at University of Bath, UK in 2001. She completed her PhD studies from King's College London, UK in 2008 in the field of Telecommunication Engineering. She is currently working at MIMOS Berhad wireless communication cluster as a staff researcher since 2009. Apart from her main task in doing research in cognitive radio, WLAN, OFDM, space-time coding, MIMO systems and wireless system, she is actively involved in the development of Malaysia technical specification, standard and guidelines of wireless terminal devices, International Mobile Telecommunication (IMT) systems and wireless sensor network for Malaysia Communications and Multimedia Commission (MCMC). She has occasionally represented Malaysia at International Telecommunication Union (ITU) working party 5D meeting for IMT-Advanced technology. Dr. Wahidah has published more than twenty refereed publications and filed several patents on her research findings. She is a member of the IEEE and senior member of IACST.

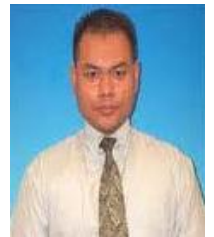

Ahmad F. Ismail is currently serving as a lecturer at the Department of Electrical and Computer Engineering, Faculty of Engineering, International Islamic University Malaysia. He completed his bachelor degree studies in Electrical Engineering at Gannon University, Pennsylvania, USA with Cum Laude Latin honors. He holds MSc in telecommunications and information systems from University of Essex, $\mathrm{UK}$ and $\mathrm{PhD}$ in Electronics from University of Bath, UK. His research interests include millimeter and microwave propagation studies, development of active and passive target tracking algorithms and Cognitive Radio (CR) applications. He is a registered Professional Engineer with Board of Engineering Malaysia and also a senior member of the IEEE.

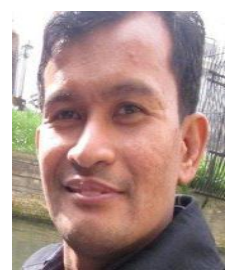

Nuzli M. Anas received the B. Eng and M. Eng degrees in Electrical Engineering from Universiti Teknologi Malaysia, Johor, in 2000 and 2013, respectively. He is currently a Senior Researcher of the Wireless Network Protocol Group, MIMOS Berhad, Kuala Lumpur, Malaysia, which he joined in 2008. He has worked as a Researcher with TM Research \& Development since 2002. His research interests include cognitive radio, wireless networking, information theory and statistical signal processing. 


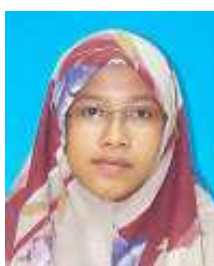

Huda Adibah Mohd Ramli completed her $\mathrm{PhD}$ at the Faculty of Engineering and Information Technology, University of Technology, Sydney (UTS), Australia. She received a M.Sc. in software engineering from University of Technology Malaysia in 2006) and

B.Eng. in electrical and computer engineering from International Islamic University Malaysia in 2003. She is now an assistant professor at Faculty of Engineering, International Islamic University Malaysia. Her current research h interests focus on radio resource management for the future wireless IP networks.

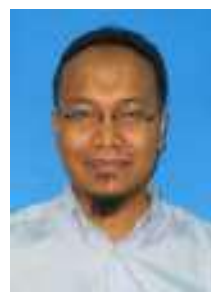

Khaizuran Abdullah obtained his BSc in electrical Eng. from University of Ohio USA in 1997 and completed his $\mathrm{ME}$ in electrical Eng. at Universiti Teknologi Malaysia in 2003. He received $\mathrm{PhD}$ in electrical and computer Eng. from RMIT University, Melbourne, Australia. He is an assistant professor at the Faculty of Engineering, International Islamic University Malaysia. His research interests are in the area of cognitive radio as well as advance modulation design. He is a senior member of IEEE and member of IEM. 